\title{
Mouth Partial Response GvHD
}

National Cancer Institute

\section{Source}

National Cancer Institute. Mouth Partial Response GVHD. NCI Thesaurus. Code

C126716.

An NIH modified Oral Mucosa Rating Scale (OMRS) score that has decreased by 2 or more points. 